\title{
Analysis of the Application of Linear Algebraic Method in Solving Higher Mathematics Problems
}

\author{
Yin Deyu \\ Public Teaching Department of Rizhao Polytechnic, Rizhao City zip code 276826, Shandong, China \\ yindeyusd@126.com
}

Keywords: Higher mathematics; Problem solving; Linear algebra.

\begin{abstract}
The education is to improve the comprehensive quality of the students and cultivate the students' cultural quality. Mathematics teaching curriculum is the main subjects of education in China through several decades of learning career. In order to improve the teaching efficiency of China's colleges and universities, many colleges and universities have carried out higher mathematics and linear algebraic methods. Therefore, in order to develop the mathematics education of China, the linear algebraic method in solving the problem of higher mathematics arises at the historic moment.
\end{abstract}

\section{Introduction}

With the development of science and technology, data analysis and other social phenomena, mathematics teaching is of great significance to the development of today's society. Higher mathematics in colleges and universities is sublimated in the traditional mathematics teaching, and its learning difficulty is greatly increased. In order to enable students to have a comprehensive understanding of mathematical problem-solving methods, so as to improve the achievement of mathematics teaching in higher learning institutions, we divide the knowledge structure under the background of higher mathematics, and linear algebra is one of them. As a branch of mathematics, linear algebra is an important part of mathematics teaching. Therefore, the learning level of college students depends largely on the advantages and disadvantages of linear algebra learning. Based on this, in order to explore the importance of the application of linear algebra to enhance the importance of higher mathematics, this paper briefly analyzes the application of linear algebra method in solving higher mathematics problems.

\section{A simple analysis of linear algebraic method}

A branch of Higher Mathematics in our country today [2], which is mainly to deal with the linear relation problem. The problem of linear relation is that each object in mathematics is expressed in one form. The most common equation is the three dimensional equation of the space plane; the two element equation in analytic geometry; in other words, the linear relation is a linear equation containing $\mathrm{N}$ unknown numbers. "Linear" mainly refers to the mathematical relation of $\mathrm{F}(\mathrm{x}+\mathrm{y})=\mathrm{f}$ (y). "Algebraic" mainly refers to the use of symbols for elements and operations kicking. Study on linear algebra is: $\mathrm{F}(\mathrm{Xy})=\mathrm{f}(\mathrm{x}) \mathrm{F}(\mathrm{y})$ linear relationship.

At the present stage, linear algebra is an important part of advanced mathematics. It has important applications in physics and technology. Therefore, linear algebra is the focus of algebra nowadays. With the continuous development of mathematics, linear algebra, the meaning has gradually expanded, and relates to the field of more extensive, not only that, the theory of linear algebra and method as well as the influence of teaching branch of mathematics, is also the indispensable basic knowledge of algebra theory physics and theoretical chemistry.

It can be seen that linear algebra is one of the important ways to reflect the essence of mathematical knowledge. With the constant attention to education in our country, the teaching process is constantly deepening reform. As an important subject in science and science, the 
application of linear algebra is more complex in the development of society. The combination of the two has a good complementarity to the mathematical problem solving. Therefore, nowadays, the development of Higher Mathematics in universities and colleges is inseparable from linear algebra. The combination of them is closely related to each other, so as to jointly promote the optimal development of mathematics teaching achievement in China [3].

\section{Analysis of the method of solving linear algebra in Higher Mathematics}

\subsection{Simplification method}

The simplified method, as the name suggests, simplifies the complex problem. In this process, we decompose, change, and change elements. as

$\mathrm{Y}=\sin 4 \mathrm{x}+\cos 4 \mathrm{x}$, $\mathrm{YN}$

When this kind of higher function is solved, the known problem is just an equation without any other hints. Therefore, the students will be confused in the process of solving the problem. In this process, it is necessary to apply the simplified method, that is,

$Y=\sin 4 x+\cos 4 x+2 \sin 2 x \cos 2 x-2 \sin 2 x \cos 2 x$

$=1-2 \sin 2 x \cos 2 \mathrm{x}$

$=1-1 / 2 \sin 22 x$

$=1-(1-\cos 4 \mathrm{x}) / 4$

So $y=3 / 4+1 / 4 \cos 4 x$ can be obtained.

$\mathrm{Yn}=4 \mathrm{n}-1 \cos (4 \mathrm{x}+\mathrm{n}$. PI /2)

Thus, the final result is obtained by simplifying the process of solving the problem. Its main purpose is to make students find ways in the process and to develop students' thinking.

\subsection{Intuitionistic analysis}

Intuitive analysis refers to finding rules, methods and conclusions through examples in order to improve students' ability of independent analysis and lay a good foundation for students to solve problems. Figure (picture excerpt network), the demand curve

The oblique progressive line of $\mathrm{F}(\mathrm{x})=\mathrm{xex} 1 / 2$

Through visual analysis, the information given by the subject is observed, and then the students are guided to be analyzed.

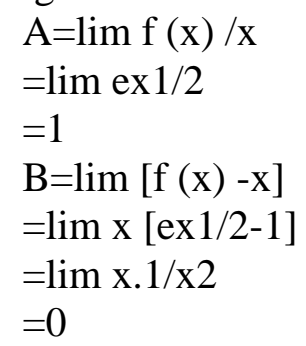

As a result, the curve has a progressive line $\mathrm{y}=\mathrm{x}$.

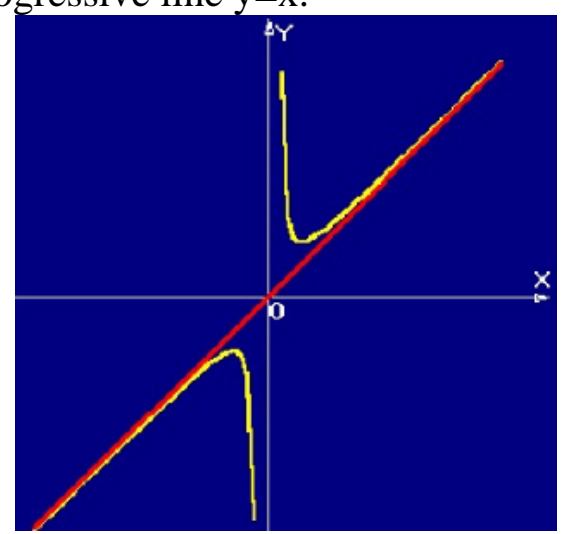




\subsection{Reverse analysis method}

Reverse analysis means the ability to exercise the reverse thinking in the process of solving the problem, and then to reverse, prove and back out. Thus, the students' thinking of solving problems is constantly exercised in the process. as

Set function $\mathrm{f}(\mathrm{x})$ on $[0,1]$ two order derivable, and $\mathrm{f}(1)=\mathrm{f}(0)$,

At least it proves that there is a point $(0,1)$, the zeta, $2 \mathrm{f}(-1,+f$, zeta) (zeta) $=0$

In order to make students enter the topic quickly, we should start with the conclusion and stimulate the students' reverse thinking. Thus can be obtained $2(x-1) f,(x)+(x-1) 2 F,(x)=[(x-1) 2 F,(x)]$,

On this basis, the auxiliary function $\mathrm{f}(\mathrm{x})=(\mathrm{x}-1) 2 \mathrm{~F},(\mathrm{x})$

In order to use $\mathrm{f}(\mathrm{x})$ satisfies the Rolle theorem in 0,1 , known by this $\mathrm{f}$ (ETA) $=0$

Then in the $\mathrm{F}(\mathrm{x})$ in the ETA, using Rolle theorem 1. Finally, the answer.

\subsection{Analogy method}

The analogy method, as the name suggests, is to find the similarity in the process of solving the problem, so that students find problems and solve problems in the process of solving the problem. Such as:

Calculation limit Lim $[1 / 1 \cdot 2.3+1 / 2.3 .4+\ldots+1 / n(n+1)(n+2)]$

Analogy limit calculation

Such as $\operatorname{Lim}[1 / 1.2+1 / 2.3+\ldots+n(n+1)]$

$=\lim [2-1 / 1.2+3-2 / 2.3+\ldots+(n+1)-n / n(n+1)]$

$=\lim [(1-1 / 2)+(1 / 2-1 / 3)+\ldots+(1 / n-1 / n+1)]$

$=\lim [1-1 / \mathrm{n}+1]$

$=1$

In order to find the final results, and in this process, the students' idea of solving the problem is gradually clear and progressive.

\subsection{Summary}

It can be seen that various methods of solving problems in linear algebra provide a good foundation for students, so as to promote students' cognition of advanced mathematics, and gradually eliminate the conflict psychology of higher mathematics, and gradually improve the traditional examination oriented thinking. Under this basis, the students' thinking of solving problems is not only promoted, but also the efficiency of students' learning.

\section{The application of linear algebra to solving the problem of Higher Mathematics}

\subsection{The application of the 1. two times theory}

In higher mathematics, the two theoretical use of linear algebra is the key content. The value of the size extremum is carried out in the real unit sphere. The complexity of advanced mathematics is mainly due to the need for many regular things and specific formulas in the curriculum, and what we need to remember is nothing less than Chinese. On this basis, the higher mathematical subjects are also required to carry out careful calculations under high intensity memory, thus finding the final results. Based on this, for linear algebra to play its greatest advantage in higher mathematics, the application of the two - type theory is very important. In the process of solving problems, the two type theory runs through the whole problem-solving process, which will make the problem-solving process gradually reasonable and standardized, so as to promote students' problem-solving efficiency and improve teaching outcomes.

\subsection{Orthogonal transform applications}

The application of orthogonal transform is to sublimate the two surface of space in higher mathematics, so as to determine the result of solving the problem and to open up the thinking of the students. Linear algebra in the traditional teaching, not the life of teaching into them, so that students 
and life are two separate individuals, so that students in the process of problem solving from the life category, students increased the difficulty of solving extended hinder students problem-solving thinking, so that students in the problem solving process is affected, the lack of problem solving confidence, to improve their learning enthusiasm and learning efficiency lead lag.

\subsection{Summary}

It can be seen that the application of linear algebra in solving problems of higher mathematics can greatly promote students' problem-solving thinking, proceed from theory to methods, and sublimate in the original concept of solving problems. In this context, in order to promote students in the process of solving the problem will not be divorced from the life category, the development of life oriented teaching is also crucial. In combination with this, the teaching achievements of higher mathematics are maximized.

\section{The application of four and two teaching methods in Algebra Teaching in Higher Mathematics}

\subsection{Straight to the point of law teaching}

One method, as the name suggests, is in each chapter are direct research teaching and learning methods of long master in the course of the study. It is not only applicable to higher mathematics, but also in some literary and liberal arts, which is self-evident in the importance of teaching based on [6]. Nowadays, the teaching achievement of higher mathematics teaching in China is lagging behind [4], which fails to achieve the expected effect. It is mainly due to the influence of exam oriented education. The traditional exam thinking problem has been deeply rooted in students' minds, and its thinking has not been explored. Higher mathematics is more complex and systematic than mathematics teaching, so that students can not find directions in the learning process, so that they can not improve their learning efficiency, which is the main factor leading to lagging behind in higher mathematics teaching. [5] Therefore, a suitable teaching method is of great significance to improve the students' traditional thinking of solving problems. Linear algebra as the difficulties present in higher mathematics, more easy to make students confused, based on this, for the development of students' thinking and improve students' learning efficiency, teaching method came into being.

\subsection{Questions Inquiry Teaching}

Problem inquiry teaching means that teachers put forward problems in the teaching class and solve them with students. Its main purpose is to stimulate students to try to solve problems in the process of exploring with teachers, and find problems in their teaching process.

The complexity of higher mathematics is obvious to all. Although the application of linear algebra makes the way of solving problems of higher students more systematic, the structure of knowledge points is still complex. Therefore, it is of great significance for students to learn how to discover problems in the process of solving problems to improve their ability to solve problems. This method will enable students to continue to open up their thinking in the process of solving problems, to emit their logical thinking ability and innovation ability. Problem inquiry teaching, when teachers ask questions, enables students to find problems in their problem-solving process gradually, so as to find out the most suitable way to solve problems, which is the embodiment of innovation ability in solving problems. So it can be seen that the problem based inquiry is based on the importance of modern higher mathematics teaching [7].

\subsection{Summary}

To sum up, to maximize the promotion of mathematics teaching achievements, not only to learn how to find a method in the problem solving process, looking for standard linear algebra applications, but also to find the accurate method of teaching. In this context, it will be combined and applied to promote the maximum achievement of higher mathematics teaching in China. 
Five, concluding remarks as the most complex and comprehensive subject in modern mathematics teaching, higher mathematics has hindered students from mastering the core of their knowledge. Therefore, the achievement of higher mathematics teaching in universities is lagging behind. The arrival of the method of solving the problem of linear algebra makes the students' thinking gradually clearer in the process of solving the problem. Thus, the application of linear algebra problem solving method has opened up a new way for college students to solve the problem. Based on this, improving the application of linear algebra problem-solving method in higher mathematics is of great significance for improving students' logical thinking ability in mathematical problem-solving. Based on this, we can improve students' learning efficiency and achieve the maximum [8] achievement in higher mathematics.

\section{References}

[1] sang Dan Dorje. Application of [J]. linear algebra methods in solving the problems of higher mathematics knowledge Herald, 2015, (7): 126-127.

[2] Lai Jingdong. The solution to the problem of higher mathematics using linear algebra method [J]. mathematics world (primary Grade 5-6 Edition), 2016, (1): 39-40.

[3] Li Decheng. Linear algebra methods in solving the problems of higher mathematics application [J]. chapter 2013, (3): 157.

[4] Wu Qiongyang. Application of linear algebraic method in solving problems of higher mathematics [J]. technology information, 2015, (11): 173.

[5] Li Xia. Several simple applications of algebraic methods in Higher Mathematics [J]. technology horizon, 2012, (17): 109-110113.

[6] Huang Xiaofei. Linear algebra methods in solving the problems of higher mathematics application of [J]. science and Technology Innovation Herald, 2015, (19): 155-155157.

[7] Zhang Shaoyan. Linear algebra methods of higher mathematics to solve problems in the application of [J]. information, 2017, (47): 224.

[8] linear algebra. Theory. Method. Application of [Z], Chongqing College of Post and Telecom,2017. 